\title{
XPS and SEM/STEM Characterization of Silver Nanoparticles Formed from the X-ray-Induced and Thermal Reduction of Silver Behenate
}

\author{
B.R. Strohmeier, ${ }^{*}$ K.L. Bunker,* C.L. Lopano,* J.P. Marquis Jr., ${ }^{*}$ J.D. Piasecki,* K.E. \\ Bennethum, ${ }^{*}$ R.G. White, ${ }^{* *}$ T. Nunney, ${ }^{* *}$ and R.J. Lee* \\ * RJ Lee Group, Inc., 350 Hochberg Road, Monroeville, PA 15146 \\ ** Thermo Fisher Scientific, Birches Industrial Est., Imberhorne La., East Grinsted, RH191UB, UK
}

This study is the first reported use of X-ray photoelectron spectroscopy (XPS or ESCA) to characterize the organometallic compound silver behenate and its X-ray-induced and thermal reduction. Silver behenate is a long-chain silver carboxylate, $\mathrm{CH}_{3}\left(\mathrm{CH}_{2}\right)_{20} \mathrm{COOAg}$, that crystallizes as a dimer in a head-to-head configuration [1-3]. Various silver compounds, including silver behenate, are used as primary components in commercially available photothermographic (PTG) and thermographic (TG) imaging elements [1-3]. Individual particles of silver behenate powder typically exist as plate-like crystals with surface dimensions of 0.2-2 $\mu \mathrm{m}$ and thicknesses of $100 \mathrm{~nm}$ or less [4,5]. Imaging in PTG and TG devices is based on the formation of silver metal nanoparticles in the 5-30 $\mathrm{nm}$ range (and larger aggregates) by the thermal reduction of silver behenate dispersed in a binder incorporated with toner and development chemistry [1-3]. Silver behenate has also found use as a standard reference material for low-angle calibration of X-ray diffraction instruments because its crystal structure produces a unique multi-peak diffraction pattern (Fig. 1) $[4,5]$.

In this study, the X-ray-induced reduction of silver behenate during exposure to monochromatic Al $\mathrm{K} \alpha \mathrm{X}$-rays in a micro-XPS instrument (Thermo Scientific K-Alpha XPS) was investigated as well as the thermal reduction at $100{ }^{\circ} \mathrm{C}$ and $250{ }^{\circ} \mathrm{C}$. In addition, combined scanning electron microscopy (SEM) and scanning transmission electron microscopy (STEM) (Hitachi S-5500 SEM/STEM) was also used in this study as a complementary technique to XPS.

Quantitative XPS analysis of silver behenate was consistent with the theoretical C:O:Ag atomic composition. Brown discoloration of silver behenate powder begins within a few seconds of exposure to $\mathrm{Al} \mathrm{K} \alpha \mathrm{X}$-rays and increases significantly with time. Noticeable changes to the XPS spectra and the observed surface composition begin to occur after about 30 minutes of X-ray exposure (Fig. 2). Prolonged exposure to Al Ka X-rays resulted in significant changes in the $\mathrm{C} 1 \mathrm{~s}$, $\mathrm{O} 1 \mathrm{~s}$, and Ag 3d peak shapes and positions. Changes in the XPS spectra indicated that exposure to $\mathrm{Al} \mathrm{K} \alpha \mathrm{X}$-rays results in the formation of silver metal particles and decomposition of the carboxylic acid potion of the molecule to hydrocarbon species. Thermal reduction of silver behenate powder produced similar changes in the XPS spectra. SEM/STEM images of silver behenate particles and silver metal nanoparticles formed by thermal reduction of silver behenate are shown in Figure 3 .

Sample heating effects during the XPS analyses should be minimal; however the ultrahigh vacuum environment and the emission of photoelectrons through the surface layers of the powder may play a role in the rapid discoloration and reduction observed in the XPS instrument. This study demonstrates that XPS and SEM/STEM are complementary techniques for investigating the chemical composition and morphology of nanomaterials. 


\section{References:}

[1] T.N. Blanton et al., Adv. X-ray Anal. 45 (2002) 371.

[2] T.N. Blanton et al., Adv. X-ray Anal. 48 (2005) 27.

[3] T.N. Blanton et al., J. Imaging Sci. Technol. 49 (2005) 356.

[4] T.N. Blanton et al., Powder Diffr. 10 (1995) 91.

[5] T.N. Blanton et al., J. Appl. Cryst. 33 (2000) 172.

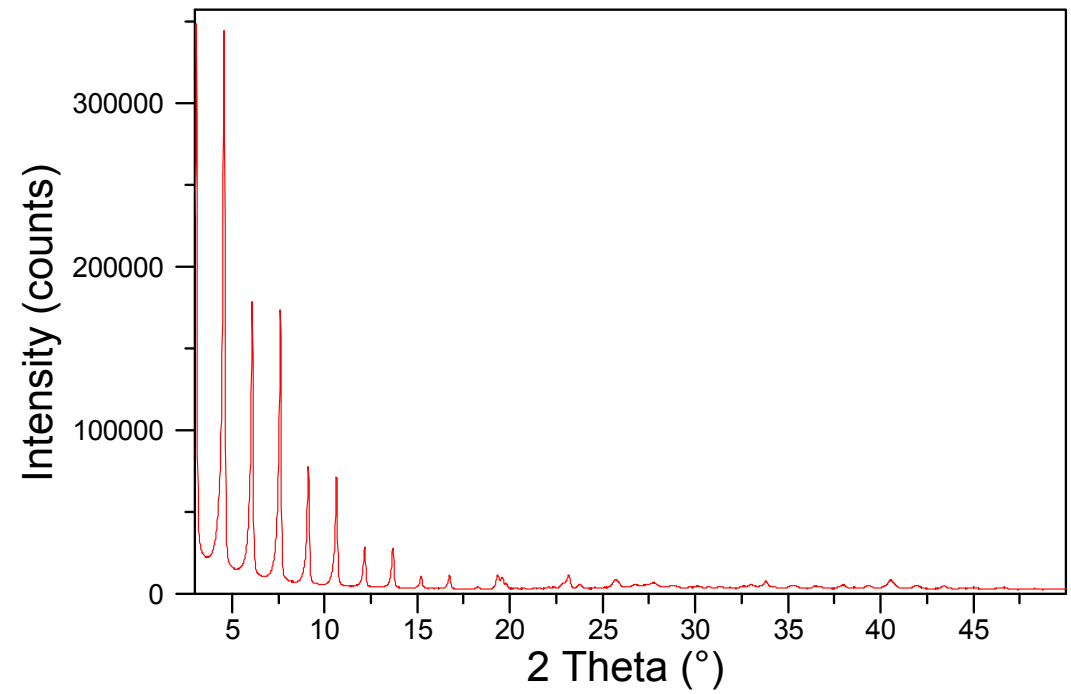

Fig. 1: X-ray diffraction pattern of silver behenate powder.

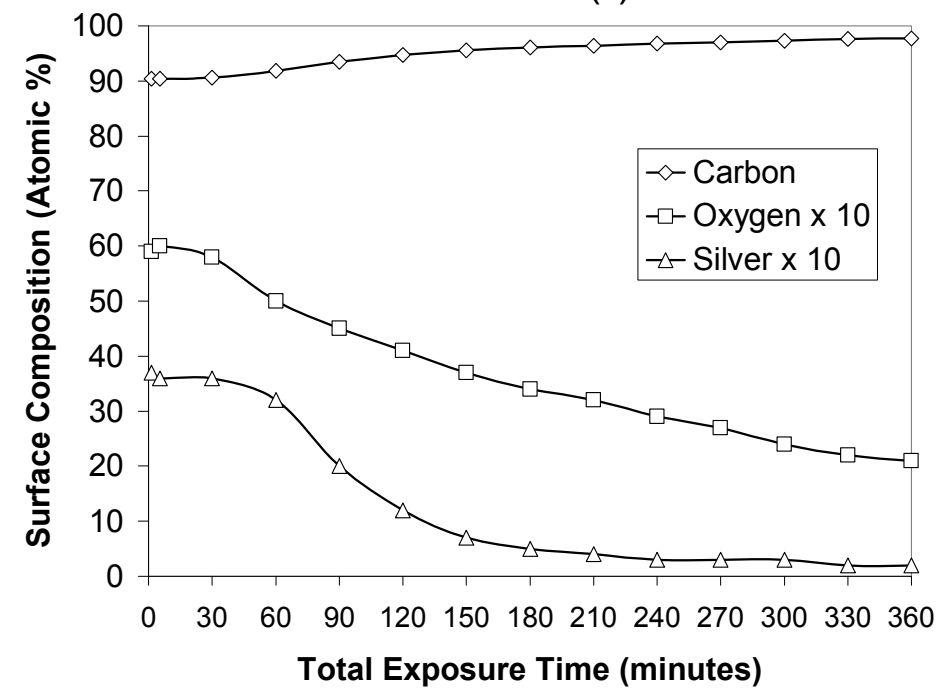

Fig. 2: Variation in surface composition of silver behenate powder with X-ray exposure time during XPS analysis.
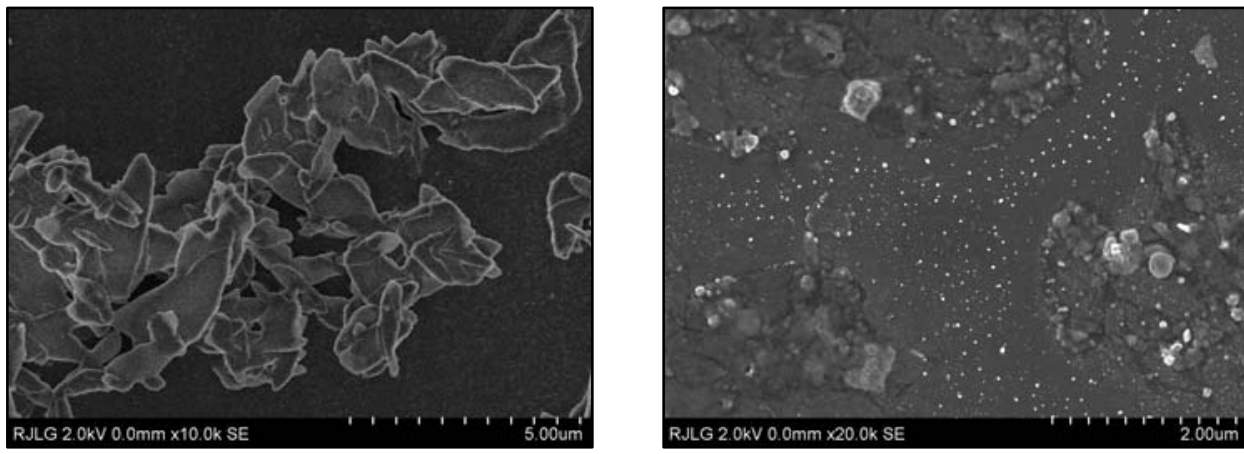

Fig. 3: Secondary electron images of (left) silver behenate particles before heating and (right) silver nanoparticles created after 20 minutes at $250{ }^{\circ} \mathrm{C}$. 